\title{
The Relativistic Transmission and Reflection Coefficients for Woods-Saxon Potential
}

\author{
B.H. YazarloO* AND H. Mehraban \\ Physics Department, Semnan University, P.O. Box 35195-363, Semnan, Iran \\ (Received August 27, 2015; in final form May 12, 2016)
}

\begin{abstract}
The relativistic problem of spin- $1 / 2$ particles subject to the Woods-Saxon potential is investigated by using the functional analysis method. We obtain scattering and bound state solutions of the one-dimensional Dirac equation with the Woods-Saxon potential in terms of the Jacobi polynomials. We also calculated the transmission and reflection coefficients by using behavior of the wave functions at infinity.
\end{abstract}

DOI: 10.12693/APhysPolA.129.1089

PACS/topics: 03.65.Nk, 03.65.Ge, 03.65.Pm

\section{Introduction}

Among the lengthy list of physical potentials, there are only few ones which yield acceptable results in particle and nuclear physics. The Woods-Saxon potential (WSP for short), which is normally considered as a mean field one, lies within this category. It has been used in the study of nuclei outside $A=110-210$ [1], high spin states in ${ }^{146} \mathrm{Gd}$ [2], parametrization of the $n^{-208} \mathrm{~Pb}$ mean field [3], two-centre formalism [4], shell model calculations [5], spectra of rotating nuclei [6], confined quantum systems [7], collective models [8] and the wobbling excitations [10]. The solution of the Klein-Gordon equation under the WSP has been obtained in [11]. By using the supersymmetry quantum mechanics the solution of twobody spinless Salpeter equation for the WSP has been reported in [12]. References [13-16] investigate Dirac equation under this interaction. Here, we intend to solve Dirac equation for the WPS and investigate the scattering and bound state solutions, and then obtain the transmission and reflection coefficients.

The outline of this paper is as follows. In the next section we present a brief review of the Dirac equation in $1+1$ dimension. Then, we present the solution of scattering states in Sect. 3 and obtain the reflection and transmission coefficients in this section. We report the bound state solution of the system under WSP in Sect. 4 . Finally, the conclusion appears in Sect. 5.

\section{Dirac equation in a $1+1$ dimension}

In $1+1$ dimension, the Dirac equation for spin- $1 / 2$ particle in the presence of vector $\left(V_{v}\right)$, scalar $\left(V_{s}\right)$ and pseudoscalar $\left(V_{p}\right)$ potentials is written as [17]:

$$
H \psi=E \psi,
$$

\footnotetext{
* corresponding author; e-mail: h. yazarloo@students.semnan.ac.ir
}

$$
\begin{aligned}
H & =\sigma_{1} p+\sigma_{3} m+\frac{\left(1+\sigma_{3}\right)}{2} \Sigma+\frac{\left(1-\sigma_{3}\right)}{2} \Delta \\
& +\sigma_{2} V_{p},
\end{aligned}
$$

where $m$ is mass of particle, and $\Sigma, \Delta$ are $V_{v}+V_{s}$ and $V_{v}-V_{s}$, respectively. $\sigma_{1}, \sigma_{2}$ and $\sigma_{3}$ denote the Pauli matrices. Considering the wave function $\psi$ as $\psi=\left(\begin{array}{c}\psi_{+} \\ \psi_{-}\end{array}\right)$, in the case of $\Delta=0$ and $E \neq-m$, the Dirac equation becomes

$$
\begin{aligned}
& \psi_{-}=-\mathrm{i} \frac{\frac{\mathrm{d} \psi_{+}}{\mathrm{d} x}-V_{p} \psi_{+}}{E+m}, \\
& -\frac{\mathrm{d}^{2} \psi_{+}}{\mathrm{d} x^{2}}+\left[(E+m) \Sigma+V_{p}^{2}+\frac{\mathrm{d} V_{p}}{\mathrm{~d} x}\right] \psi_{+}= \\
& \left(E^{2}-m^{2}\right) \psi_{+} .
\end{aligned}
$$

For $\Sigma=0$ and $E \neq m$, we can obtain the Dirac equation from the previous ones by doing $\psi_{+} \leftrightarrow \psi_{-}, m \rightarrow$ $-m, \Sigma \rightarrow \Delta$ and $V_{p} \rightarrow-V_{p}$.

\section{Scattering states of Dirac equation for Woods-Saxon potential}

We consider the pseudoscalar $\left(V_{p}\right)$ and $\Sigma$ potentials as the Woods-Saxon potential [18]:

$$
\begin{aligned}
& \Sigma=V_{0}\left[\frac{\theta(-x)}{\xi}+\frac{\theta(x)}{\tilde{\xi}}\right], \\
& V_{p}=V_{1}\left[\frac{\theta(-x)}{\xi}+\frac{\theta(x)}{\tilde{\xi}}\right],
\end{aligned}
$$

where $V_{0}$ and $V_{1}$ are the strengths of potential, $\xi=$ $1+q \mathrm{e}^{\frac{-x-R}{a}}$ and $\tilde{\xi}=1+\tilde{q} \mathrm{e}^{\frac{-x-R}{a}} \cdot \tilde{q}$ and $q$ are deformation parameters. As we are investigating the scattering states of the equation, we first consider $x<0$. Substitution of Eqs. (4a) and (4b) in Eq. (3) allows us to obtain

$$
\frac{\mathrm{d}^{2} \psi_{n+}^{L}(x)}{\mathrm{d} x^{2}}-\left[\frac{V_{0}\left(E_{n}+m\right)}{\xi}+\frac{V_{1}^{2}}{\xi^{2}}+\frac{q V_{1}}{a} \frac{\mathrm{e}^{\frac{-x-R}{a}}}{\xi^{2}}\right]
$$




$$
\times \psi_{n+}^{L}(x)=-\left(E^{2}-m^{2}\right) \psi_{n+}^{L}(x) .
$$

Applying the two transformations of the form

$$
\begin{aligned}
& y_{L}=-x-R, \alpha=\frac{1}{a}, \\
& z_{L}=\frac{1}{1+q \mathrm{e}^{\alpha y_{L}}},
\end{aligned}
$$

Eq. (5) takes the form

$$
\begin{aligned}
& \frac{\mathrm{d}^{2} \psi_{n+}^{L}\left(z_{L}\right)}{\mathrm{d} z_{L}^{2}}+\frac{\left(1-2 z_{L}\right)}{z_{L}\left(1-z_{L}\right)} \frac{\mathrm{d} \psi_{n+}^{L}\left(z_{L}\right)}{\mathrm{d} z_{L}} \\
& +\frac{1}{z_{L}^{2}\left(1-z_{L}\right)^{2}}\left\{-\frac{1}{\alpha^{2}}\left(V_{1}^{2}-\alpha V_{1}\right) z_{L}^{2}\right. \\
& \left.+\frac{1}{\alpha^{2}}\left[-V_{0}\left(E_{n}+m\right)-\alpha V_{1}\right] z_{L}-\frac{1}{\alpha^{2}}\left(m^{2}-E^{2}\right)\right\} \\
& \times \psi_{n+}^{L}\left(z_{L}\right)=0 .
\end{aligned}
$$

Therefore, the solution of Eq. (8) can be expressed as

$$
\begin{aligned}
& \psi_{n+}^{L}(x)=A\left(\frac{1}{\xi}\right)^{\mathrm{i} \sqrt{-w}}\left(1-\frac{1}{\xi}\right)^{\sqrt{g_{L}-h_{L}+w}} \\
& \times P_{n}^{\left(2 \sqrt{w}, 2 \sqrt{g_{L}-h_{L}+w}\right)}\left(1-\frac{2}{\xi}\right) \\
& +B\left(\frac{1}{\xi}\right)^{-\mathrm{i} \sqrt{-w}}\left(1-\frac{1}{\xi}\right)^{\sqrt{g_{L}-h_{L}+w}} \\
& \times P_{n}^{\left(-2 \sqrt{w}, 2 \sqrt{g_{L}-h_{L}+w}\right)}\left(1-\frac{2}{\xi}\right),
\end{aligned}
$$

which for the limit $x \rightarrow-\infty\left(z_{L} \rightarrow 0\right.$ and $\left.\left(1-z_{L}\right)^{\sqrt{g_{L}-h_{L}+w}} \rightarrow 1\right)$ :

$$
\begin{gathered}
\psi_{n+}^{L}(x \rightarrow-\infty) \propto A\left(\frac{1}{\xi}\right)^{\mathrm{i} \sqrt{-w}}+B\left(\frac{1}{\xi}\right)^{-\mathrm{i} \sqrt{-w}} \\
\simeq A\left(\frac{1}{q \mathrm{e}^{\frac{-x-R}{a}}}\right)^{\mathrm{i} \sqrt{-w}}+B\left(\frac{1}{q \mathrm{e}^{\frac{-x-R}{a}}}\right)^{-\mathrm{i} \sqrt{-w}}= \\
A q^{-\sqrt{w}} \mathrm{e}^{-\mathrm{i} \sqrt{-w}\left(\frac{-x-R}{a}\right)}+B q^{\sqrt{w}} \mathrm{e}^{\mathrm{i} \sqrt{-w}\left(\frac{-x-R}{a}\right)},
\end{gathered}
$$

and parameters defined in Eq. (9) have the following form:

$$
\begin{aligned}
& g_{L}=\frac{1}{\alpha^{2}}\left(V_{1}^{2}-\alpha V_{1}\right), h_{L}=\frac{1}{\alpha^{2}}\left(-V_{0}\left(E_{n}+m\right)-\alpha V_{1}\right), \\
& w=\frac{1}{\alpha^{2}}\left(m^{2}-E_{n}^{2}\right) .
\end{aligned}
$$

Here, we study the solution for region $x>0$, by inserting Eqs. (4a) and (4b) in Eq. (3), we have

$$
\begin{aligned}
& \frac{\mathrm{d}^{2} \psi_{n+}^{R}(x)}{\mathrm{d} x^{2}}-\left[\frac{V_{0}\left(E_{n}+m\right)}{\tilde{\xi}}+\frac{V_{1}^{2}}{\tilde{\xi}^{2}}-\frac{\tilde{q} V_{1}}{a} \frac{\mathrm{e}^{\frac{x-R}{a}}}{\tilde{\xi}^{2}}\right] \\
& \times \psi_{n+}^{R}(x)=-\left(E^{2}-m^{2}\right) \psi_{n+}^{R}(x) .
\end{aligned}
$$

Now, rewriting Eq. (12) by employing the convenient transformations $y_{R}=x-R, \alpha=\frac{1}{a}$ and $z_{R}=$ $\frac{1}{\left(1+\tilde{q} \mathrm{e}^{\left.\alpha y_{R}\right)}\right.}$ we find the following simple hypergeometric form given by:

$$
\begin{aligned}
& \psi_{n+}^{R}(x)=C\left(\frac{1}{\tilde{\xi}}\right)^{\mathrm{i} \sqrt{-w}}\left(1-\frac{1}{\tilde{\xi}}\right)^{\sqrt{g_{R}-h_{R}+w}} \\
& \times P_{n}^{\left(2 \sqrt{w}, 2 \sqrt{g_{R}-h_{R}+w}\right)}\left(1-\frac{2}{\tilde{\xi}}\right) \\
& +D\left(\frac{1}{\tilde{\xi}}\right)^{-\mathrm{i} \sqrt{-w}}\left(1-\frac{1}{\tilde{\xi}}\right)^{\sqrt{g_{R}-h_{R}+w}} \\
& \times P_{n}^{\left(-2 \sqrt{w}, 2 \sqrt{g_{R}-h_{R}+w}\right)}\left(1-\frac{2}{\tilde{\xi}}\right) .
\end{aligned}
$$

In order to define a plane wave travelling from left to right we have to set $C=0$, so we have

$$
\begin{aligned}
& \psi_{n+}^{R}(x)=D\left(\frac{1}{\tilde{\xi}}\right)^{-\mathrm{i} \sqrt{-w}}\left(1-\frac{1}{\tilde{\xi}}\right)^{\sqrt{g_{R}-h_{R}+w}} \\
& \times P_{n}^{\left(-2 \sqrt{w}, 2 \sqrt{g_{R}-h_{R}+w}\right)}\left(1-\frac{2}{\tilde{\xi}}\right),
\end{aligned}
$$

where

$$
\begin{aligned}
& g_{R}=\frac{1}{\alpha^{2}}\left(V_{1}^{2}+\alpha V_{1}\right), h_{R}=\frac{1}{\alpha^{2}}\left(-V_{0}\left(E_{n}+m\right)+\alpha V_{1}\right), \\
& w=\frac{1}{\alpha^{2}}\left(m^{2}-E_{n}^{2}\right) .
\end{aligned}
$$

In limit of $x \rightarrow \infty\left(z_{R} \rightarrow 0\right.$ and $\left.\left(1-z_{R}\right)^{\sqrt{g_{R}-h_{R}+w}} \rightarrow 1\right)$, Eq. (14) becomes

$$
\begin{gathered}
\psi_{n+}^{R}(x \rightarrow \infty) \propto D\left(\frac{1}{\tilde{\xi}}\right)^{-\mathrm{i} \sqrt{-w}} \simeq D\left(\frac{1}{\tilde{q} \mathrm{e}^{\frac{x-R}{a}}}\right)^{-\mathrm{i} \sqrt{-w}}= \\
D \tilde{q}^{\sqrt{w}} \mathrm{e}^{\mathrm{i} \sqrt{-w}\left(\frac{x-R}{a}\right)} .
\end{gathered}
$$

As a result we can summarize the wave function for the limit $x \rightarrow \pm \infty$ from Eq. (10) and Eq. (16) as

$$
\psi(x)=\left\{\begin{array}{c}
A q^{-\sqrt{w}} \mathrm{e}^{-\mathrm{i} \sqrt{-w}\left(\frac{-x-R}{a}\right)} \\
+B q^{\sqrt{w}} \mathrm{e}^{\mathrm{i} \sqrt{-w}\left(\frac{-x-R}{a}\right)} \\
x \rightarrow-\infty \\
D \tilde{q}^{\sqrt{w}} \mathrm{e}^{\mathrm{i} \sqrt{-w}\left(\frac{x-R}{a}\right)} \\
x \rightarrow \infty,
\end{array}\right.
$$

and the value of $A, B$ and $D$ can be found from the continuity conditions, i.e. $\psi_{n+}^{L}(x=0)=\psi_{n+}^{R}(x=0)$ and $\left.\psi_{n+}^{\prime} L(x=0)=\psi_{n+}^{\prime} R=0\right)$, so we have

$$
\begin{aligned}
& A \xi_{L}^{\beta}\left(1-\xi_{L}\right)^{\gamma_{L}} G_{1}+B \xi_{L}^{-\beta}\left(1-\xi_{L}\right)^{\gamma_{L}} G_{2}= \\
& \quad D \xi_{R}^{-\beta}\left(1-\xi_{R}\right)^{\gamma_{R}} G_{3}, \\
& A\left[\frac{\beta q}{a} \mathrm{e}^{-\frac{R}{a}} \xi_{L}^{\beta+1}\left(1-\xi_{L}\right)^{\gamma_{L}} G_{1}-\frac{\gamma_{L} q}{a} \mathrm{e}^{-\frac{R}{a} \xi_{L}^{\beta+2}}\right. \\
& \quad \times\left(1-\xi_{L}\right)^{\gamma_{L}-1} G_{1}-\frac{q}{a}\left(2 \sqrt{w}+2 \sqrt{g_{L}-h_{L}+w}\right. \\
& \left.\quad+n+1) \mathrm{e}^{-\frac{R}{a}} \xi_{L}^{\beta+1}\left(1-\xi_{L}\right)^{\gamma_{L}}\left(G_{1}-G_{4}\right)\right] \\
& \quad+B\left[-\frac{\beta q}{a} \mathrm{e}^{-\frac{R}{a}} \xi_{L}^{-\beta+1}\left(1-\xi_{L}\right)^{\gamma_{L}} G_{2}\right.
\end{aligned}
$$




$$
\begin{aligned}
& -\frac{\gamma_{L} q}{a} \mathrm{e}^{-\frac{R}{a}} \xi_{L}^{-\beta+2}\left(1-\xi_{L}\right)^{\gamma_{L}-1} G_{2}-\frac{q}{a}(-2 \sqrt{w} \\
& \left.+2 \sqrt{g_{L}-h_{L}+w}+n+1\right) \mathrm{e}^{-\frac{R}{a}} \xi_{L}^{-\beta+1}\left(1-\xi_{L}\right)^{\gamma_{L}} \\
& \left.\times\left(G_{2}-G_{5}\right)\right]=D\left[\frac{\beta \tilde{q}}{a} \mathrm{e}^{-\frac{R}{a}} \xi_{R}^{-\beta+1}\left(1-\xi_{R}\right)^{\gamma_{R}} G_{3}\right. \\
& +\frac{\gamma_{R} \tilde{q}}{a} \mathrm{e}^{-\frac{R}{a}} \xi_{R}^{-\beta+2}\left(1-\xi_{R}\right)^{\gamma_{R}-1} G_{3}+\frac{\tilde{q}}{a}(-2 \sqrt{w} \\
& \left.+2 \sqrt{g_{R}-h_{R}+w}+n+1\right) \mathrm{e}^{-\frac{R}{a}} \xi_{R}^{-\beta+1} \\
& \left.\times\left(1-\xi_{R}\right)^{\gamma_{R}}\left(G_{3}-G_{6}\right)\right]
\end{aligned}
$$

where $\beta=\mathrm{i} \sqrt{-w}, \xi_{R}=\frac{1}{1+\tilde{q} \mathrm{e}^{-\frac{R}{a}}}, \xi_{L}=\frac{1}{1+q \mathrm{e}^{-\frac{R}{a}}}$, $\gamma_{R}=\sqrt{g_{R}-h_{R}+w}, \gamma_{L}=\sqrt{g_{L}-h_{L}+w}$,

$$
\begin{aligned}
& G_{1}=P_{n}^{\left(2 \sqrt{w}, 2 \sqrt{g_{L}-h_{L}+w}\right)}\left(1-\frac{2}{1+q \mathrm{e}^{\frac{-R}{a}}}\right), \\
& G_{2}=P_{n}^{\left(-2 \sqrt{w}, 2 \sqrt{g_{L}-h_{L}+w}\right)}\left(1-\frac{2}{1+q \mathrm{e}^{\frac{-R}{a}}}\right), \\
& G_{3}=P_{n}^{\left(-2 \sqrt{w}, 2 \sqrt{g_{R}-h_{R}+w}\right)}\left(1-\frac{2}{1+\tilde{q} \mathrm{e}^{\frac{-R}{a}}}\right), \\
& G_{4}=P_{n}^{\left(2 \sqrt{w}, 2 \sqrt{g_{L}-h_{L}+w}+1\right)}\left(1-\frac{2}{1+q \mathrm{e}^{\frac{-R}{a}}}\right), \\
& G_{5}=P_{n}^{\left(-2 \sqrt{w}, 2 \sqrt{g_{L}-h_{L}+w}+1\right)}\left(1-\frac{2}{1+q \mathrm{e}^{\frac{-R}{a}}}\right), \\
& G_{6}=P_{n}^{\left(-2 \sqrt{w}, 2 \sqrt{g_{R}-h_{R}+w}+1\right)}\left(1-\frac{2}{1+\tilde{q} \mathrm{e}^{\frac{-R}{a}}}\right) .
\end{aligned}
$$

From Eq. (18) and Eq. (19), one can obtain

$$
\begin{aligned}
& \frac{B}{A}=\frac{F_{1}^{\prime} F_{2}-F_{1} F_{2}^{\prime}}{F_{1} F_{3}^{\prime}-F_{1}^{\prime} F_{3}}, \\
& \frac{D}{A}=\frac{F_{3}^{\prime}}{F_{1}^{\prime}} \frac{F_{1}^{\prime} F_{2}-F_{1} F_{2}^{\prime}}{F_{1} F_{3}^{\prime}-F_{1}^{\prime} F_{3}}+\frac{F_{2}^{\prime}}{F_{1}^{\prime}},
\end{aligned}
$$

and

$$
\begin{aligned}
F_{1} & =\frac{\beta \tilde{q}}{a} \mathrm{e}^{-\frac{R}{a}} \xi_{R}^{-\beta+1}\left(1-\xi_{R}\right)^{\gamma_{R}} G_{3} \\
& +\frac{\gamma_{R} \tilde{q}}{a} \mathrm{e}^{-\frac{R}{a}} \xi_{R}^{-\beta+2}\left(1-\xi_{R}\right)^{\gamma_{R}-1} G_{3} \\
& +\frac{\tilde{q}}{a}\left(-2 \sqrt{w}+2 \sqrt{g_{R}-h_{R}+w}+n+1\right) \\
& \times \mathrm{e}^{-\frac{R}{a}} \xi_{R}^{-\beta+1}\left(1-\xi_{R}\right)^{\gamma_{R}}\left(G_{3}-G_{6}\right), \\
F_{2} & =\frac{\beta q}{a} \mathrm{e}^{-\frac{R}{a}} \xi_{L}^{\beta+1}\left(1-\xi_{L}\right)^{\gamma_{L}} G_{1} \\
& -\frac{\gamma_{L} q}{a} \mathrm{e}^{-\frac{R}{a}} \xi_{L}^{\beta+2}\left(1-\xi_{L}\right)^{\gamma_{L}-1} G_{1}
\end{aligned}
$$

$$
\begin{aligned}
& -\frac{q}{a}\left(2 \sqrt{w}+2 \sqrt{g_{L}-h_{L}+w}+n+1\right) \\
& \times \mathrm{e}^{-\frac{R}{a}} \xi_{L}^{\beta+1}\left(1-\xi_{L}\right)^{\gamma_{L}}\left(G_{1}-G_{4}\right), \\
& F_{3}=-\frac{\beta q}{a} \mathrm{e}^{-\frac{R}{a}} \xi_{L}^{-\beta+1}\left(1-\xi_{L}\right)^{\gamma_{L}} G_{2} \\
& -\frac{\gamma_{L} q}{a} \mathrm{e}^{-\frac{R}{a}} \xi_{L}^{-\beta+2}\left(1-\xi_{L}\right)^{\gamma_{L}-1} G_{2} \\
& -\frac{q}{a}\left(-2 \sqrt{w}+2 \sqrt{g_{L}-h_{L}+w}+n+1\right) \\
& \times \mathrm{e}^{-\frac{R}{a}} \xi_{L}^{-\beta+1}\left(1-\xi_{L}\right)^{\gamma_{L}}\left(G_{2}-G_{5}\right), \\
& F_{1}^{\prime}=\xi_{R}^{-\beta}\left(1-\xi_{R}\right)^{\gamma_{R}} G_{3}, F_{2}^{\prime}=\xi_{L}^{\beta}\left(1-\xi_{L}\right)^{\gamma_{L}} G_{1}, \\
& F_{3}^{\prime}=\xi_{L}^{-\beta}\left(1-\xi_{L}\right)^{\gamma_{L}} G_{2} .
\end{aligned}
$$

For $x<0$, we have $\psi_{n+}^{L}(x)=\psi_{n+\text {,inc. }}+\psi_{n+\text {,ref., }}$, and for $x>0, \psi_{n+}^{R}(x)=\psi_{n+\text {,trans. }}(x)$. The incoming, reflected and transmitted fluxes are $\boldsymbol{J}_{\text {inc. }}=\bar{\psi}_{\text {inc. }} \gamma^{\mu} \psi_{\text {inc. }}$, $\boldsymbol{J}_{\text {ref. }}=\bar{\psi}_{\text {ref. }} \gamma^{\mu} \psi_{\text {ref. }}$ and $\boldsymbol{J}_{\text {trans. }}=\bar{\psi}_{\text {trans. }} \gamma^{\mu} \psi_{\text {trans. }}$, respectively. Therefore, the current density for the left and right hand sides respectively are $\boldsymbol{J}_{L}=\boldsymbol{J}_{\text {inc. }}+\boldsymbol{J}_{\text {ref. and }}$ $\boldsymbol{J}_{R}=\boldsymbol{J}_{\text {trans. }}$. In summary, the reflection and transmission coefficients are

$$
\begin{aligned}
& R=\frac{\boldsymbol{J}_{\text {ref. }}}{\boldsymbol{J}_{\text {inc. }}}=\left|\frac{B}{A}\right|^{2}, \\
& T=\frac{\boldsymbol{J}_{\text {trans. }}}{\boldsymbol{J}_{\text {inc. }}}=\left|\frac{D}{A}\right|^{2} .
\end{aligned}
$$

\section{Bound-state solutions}

In this section, we tend to find the bound states for the Woods-Saxon potential well which means $V_{0} \rightarrow$ $-V_{0}, V_{1} \rightarrow-V_{1}$ in Eq. (4a) and Eq. (4b). The wave functions for the bound state solutions of WSP for $x<0$ and $x>0$ regions, respectively, are

$$
\begin{aligned}
& \psi_{n+}^{L}(x)=A\left(\frac{1}{\xi}\right)^{\mathrm{i} \sqrt{-w}}\left(1-\frac{1}{\xi}\right)^{\sqrt{g_{L}^{\prime}-h_{L}^{\prime}+w}} \\
& \times P_{n}^{\left(2 \sqrt{w}, 2 \sqrt{g_{L}^{\prime}-h_{L}^{\prime}+w}\right)}\left(1-\frac{2}{\xi}\right), \\
& \psi_{n+}^{R}(x)=C\left(\frac{1}{\tilde{\xi}}\right)^{\mathrm{i} \sqrt{-w}}\left(1-\frac{1}{\tilde{\xi}}\right)^{\sqrt{g_{R}^{\prime}-h_{R}^{\prime}+w}} \\
& \times P_{n}^{\left(2 \sqrt{w}, 2 \sqrt{g_{R}^{\prime}-h_{R}^{\prime}+w}\right)}\left(1-\frac{2}{\tilde{\xi}}\right),
\end{aligned}
$$

where

$$
\begin{aligned}
& g_{L}^{\prime}=\frac{1}{\alpha^{2} q^{2}}\left(V_{1}^{2}+\frac{V_{1}}{a}\right), \\
& h_{L}^{\prime}=\frac{1}{\alpha^{2} q^{2}}\left[V_{0}\left(E_{n}+m\right)+\frac{V_{1}}{a}\right],
\end{aligned}
$$




$$
\begin{aligned}
& g_{R}^{\prime}=\frac{1}{\alpha^{2} \tilde{q}^{2}}\left(V_{1}^{2}-\frac{V_{1}}{a}\right), \\
& h_{R}^{\prime}=\frac{1}{\alpha^{2} \tilde{q}^{2}}\left[V_{0}\left(E_{n}+m\right)-\frac{V_{1}}{a}\right] .
\end{aligned}
$$

By using the boundary conditions of the wave function and its derivative on Eq. (29) and Eq. (30), we may write

$$
\begin{aligned}
& A \xi_{L}^{\beta}\left(1-\xi_{L}\right)^{\gamma_{L}^{\prime}} G_{1}^{\prime}=C \xi_{R}^{\beta}\left(1-\xi_{R}\right)^{\gamma_{R}^{\prime}} G_{2}^{\prime}, \\
& A\left[\frac{\beta q}{a} \mathrm{e}^{-\frac{R}{a}} \xi_{L}^{\beta+1}\left(1-\xi_{L}\right)^{\gamma_{L}^{\prime}} G_{1}^{\prime}-\frac{\gamma_{L}^{\prime} q}{a} \mathrm{e}^{-\frac{R}{a}} \xi_{L}^{\beta+2}\right. \\
& \quad \times\left(1-\xi_{L}\right)^{\gamma_{L}^{\prime}-1} G_{1}^{\prime}-\frac{q}{a}\left(2 \sqrt{w}+2 \sqrt{g_{L}^{\prime}-h_{L}^{\prime}+w}\right. \\
& \left.\quad+n+1) \mathrm{e}^{-\frac{R}{a}} \xi_{L}^{\beta+1}\left(1-\xi_{L}\right)^{\gamma_{L}^{\prime}}\left(G_{1}^{\prime}-G_{4}^{\prime}\right)\right]= \\
& \quad C\left[-\frac{\beta \tilde{q}}{a} \mathrm{e}^{-\frac{R}{a}} \xi_{R}^{\beta+1}\left(1-\xi_{R}\right)^{\gamma_{R}^{\prime}} G_{2}^{\prime}+\frac{\gamma_{R}^{\prime} \tilde{q}}{a}\right. \\
& \quad \times \mathrm{e}^{-\frac{R}{a}} \xi_{R}^{\beta+2}\left(1-\xi_{R}\right)^{\gamma_{R}^{\prime}-1} G_{2}^{\prime} \\
& \quad+\frac{\tilde{q}}{a}\left(2 \sqrt{w}+2 \sqrt{g_{R}^{\prime}-h_{R}^{\prime}+w}+n+1\right) \mathrm{e}^{-\frac{R}{a}} \xi_{R}^{\beta+1} \\
& \left.\quad \times\left(1-\xi_{R}\right)^{\gamma_{R}^{\prime}}\left(G_{2}^{\prime}-G_{6}^{\prime}\right)\right],
\end{aligned}
$$

where $\gamma_{R}^{\prime}=\sqrt{g_{R}^{\prime}-h_{R}^{\prime}+w}, \gamma_{L}^{\prime}=\sqrt{g_{L}^{\prime}-h_{L}^{\prime}+w}$,

$$
\begin{aligned}
& G_{1}^{\prime}=P_{n}^{\left(2 \sqrt{w}, 2 \sqrt{g_{L}^{\prime}-h_{L}^{\prime}+w}\right)}\left(1-\frac{2}{1+q \mathrm{e}^{\frac{-R}{a}}}\right), \\
& G_{2}^{\prime}=P_{n}^{\left(2 \sqrt{w}, 2 \sqrt{g_{R}^{\prime}-h_{R}^{\prime}+w}\right)}\left(1-\frac{2}{1+\tilde{q} \mathrm{e}^{\frac{-R}{a}}}\right), \\
& G_{4}^{\prime}=P_{n}^{\left(2 \sqrt{w}, 2 \sqrt{g_{l}^{\prime}-h_{l}^{\prime}+w}+1\right)}\left(1-\frac{2}{1+q \mathrm{e}^{\frac{-R}{a}}}\right), \\
& G_{6}^{\prime}=P_{n}^{\left(2 \sqrt{w}, 2 \sqrt{g_{R}^{\prime}-h_{R}^{\prime}+w}+1\right)}\left(1-\frac{2}{1+\tilde{q} \mathrm{e}^{\frac{-R}{a}}}\right) .
\end{aligned}
$$

Using Eqs. (32) and (33), we can simply determine the energy eigenvalues.

\section{Conclusions}

In this research work, we considered the scattering and the bound solutions of one-dimensional Dirac equation with the Woods-Saxon potential. By using the behavior of the wave function at infinity and the continuity conditions of the wave functions we obtained the transmission and reflection coefficients. Finally, we presented the Bound states solution of Dirac equation under WoodsSaxon potential.

\section{Acknowledgments}

It is a great pleasure for authors to thank the referee for his/her many useful comments on the manuscript.

\section{References}

[1] J. Dudek, Z. Szymanski, T. Werner, A. Faessler, C. Lima, Phys. Rev. C 26, 1712 (1982).

[2] J. Dudek, A. Majhofer, J. Skalski, T. Werner, S. Cwiok, W. Nazarewicz, J. Phys. G Nucl. Particle Phys. 5, 1359 (1979).

[3] C. Mahaux, R. Sartoe, Nucl. Phys. A 468, 193 (1987).

[4] S. Cwiok, J. Dudek, W. Nazarewicz, J. Skalski, T. Werner, Comput. Sci. Commun. 46, 379 (1987).

[5] R. Bengtsson, J. Dudek, W. Nazarewicz, P. Olanders, , Phys. Scr. 39, 196 (1989).

[6] H. Molique, J. Dudek, Phys. Rev. C 56, 1795 (1997).

[7] L.S. Costa, F.V. Prudente, P.H. Acioli, J.J. Soares Neto, J.D.M. Vianna, J. Phys. B At. Mol. Opt. Phys. 32, 2461 (1999).

[8] I. Hamamoto, Phys. Rev. C 73, 064308 (2006).

[9] N. Schwierz, I. Wiedenhover, A. Volya, arXiv:0709.3525arXiv:0709.3525.

[10] T. Shoji, Y.R. Shimizu, Prog. Theor. Phys. 121, 319 (2009).

[11] C. Rojas, V.M. Villalba, Phys. Rev. A 71, 052101 (2005).

[12] S. Hassanabadi, M. Ghominejad, B.H. Yazarloo, S. Zarrinkamar, H. Hassanabadi, Chin. Phys. C 37, 083102 (2013).

[13] O. Aydoğdu, R. Sever, Eur. Phys. J. A 43, 73 (2010).

[14] G. Jian-You, F.X. Zheng, X. Fu-Xin, Phys. Rev. A 66, 062105 (2002)

[15] T. S. Chen, H.F. Lü, J. Meng, S.G. Zhou Chin. Phys. Lett. 20, 358 (2003).

[16] P. Kennedy, J. Phys. A Math. Gen. 35, 689 (2002).

[17] L.B. Castro, A.S. De Castro, M. Hott, Int. J. Mod. Phys. E 16, 3002 (2007).

[18] H. Hassanabadi, E. Maghsoodi, S. Zarrinkamar, N. Salehi, Few-Body Syst. 54, 2009 (2013). 